Y. Kitaoka

Nagoya Math. J.

Vol. 52 (1973), 147-161

\title{
QUATERNARY EVEN POSITIVE DEFINITE QUADRATIC FORMS OF PRIME DISCRIMINANT
}

\author{
YOSHIYUKI KITAOKA
}

In this note we study quaternary even positive definite quadratic forms of prime discriminant. In $\S 1$ we classify quaternary even positive definite quadratic forms of prime discriminant $p \equiv 1 \bmod 4$ (called simply nice quaternary lattices in this note) which represent two. We note that the class number of such forms is closely related to the dimension of the space of certain automorphic forms. (Remark 4 in the text). By using the classification in $\S 1$ and the theory of integral representations of cyclic groups we show that the orthogonal group of a nice quaternary lattice is generated by \pm 1 and symmetries (of the lattice). In $\S 3$, we calculate the class number of nice quaternary lattices. Notations and terminologies will generally be those of O'Meara [5]. Any exceptions to this convention will be stated explicitly. Through this note $Q(x)$ and $B(x, y)$ denote quadratic forms and corresponding bilinear forms (i.e., $2 B(x, y)=Q(x+y)-Q(x)-Q(y)$ ), and $p$ denotes a fixed prime number $\equiv 1 \bmod 4$.

§1. We say that a quadratic lattice $N$ over the ring of rational integers $Z$ is even if and only if $Q(x) \equiv 0 \bmod 2$ for any element in $N$. For brevity, a quadratic lattice $N$ is called nice in this note if and only if $N$ is an even positive definite quadratic lattice over $Z$, its discriminant $d(N)$ is $p, 2 p$ or $4 p$ according as $N$ is quarternary, ternary or binary respectively, and moreover the Hasse invariant $S_{2}(N)$ at the prime two of $N$ is $-\left(\frac{2}{p}\right)$ when $N$ is binary.

1.1. Let $L$ be a nice quaternary lattice and have an element $e_{1}$ with $Q\left(e_{1}\right)=2$. Then we can take a basis $\left\{e_{1}, e_{2}, e_{3}, e_{4}\right\}$ of $L$ satisfying $B\left(e_{1}, e_{2}\right)$ $=1$ and $B\left(e_{1}, e_{3}\right)=B\left(e_{1}, e_{4}\right)=0$ so that $Z\left[e_{1}\right]^{\perp}=Z\left[e_{1}-2 e_{2}, e_{3}, e_{4}\right]$ and $\left[L ; Z\left[e_{1}\right] \perp Z\left[e_{1}\right]^{\perp}\right]=2$. This implies that $Z\left[e_{1}\right]^{\perp}$ is a nice ternary lattice.

Received July 9, 1973. 
1.2. Lemma. Let $K$ be a nice ternary lattice. Then a maximal even quadratic lattice $L$ containing $Z\left[e_{1}\right] \perp K,\left(Q\left(e_{1}\right)=2\right)$, is uniquely determined and nice, and $Z\left[e_{1}\right]^{\perp}$ in $L$ is equal to $K$.

Proof. Since $K \cong\langle A(2 a, 2 a)\rangle \perp\langle 2 u p\rangle$ over $Z_{2}$, where $a=0$ or 1 , and $u=-1$ or 3 respectively, we have $Z\left[e_{1}\right] \perp K \cong\langle 2\rangle \perp\langle 2 u p\rangle \perp$ $\langle A(2 a, 2 a)\rangle$ over $Z_{2}$. On the other hand $\langle 2\rangle \perp\langle 2 u p\rangle$ has norm 2, but it is not maximal over $Z_{2}$. This means that $Z\left[e_{1}\right] \perp K$ is not maximal and $\left[L ; Z\left[e_{1}\right] \perp K\right]=2$, and $L$ is nice. Put $L=Z\left[e_{1}\right] \perp K+Z[u]$, where $u=\frac{1}{2}\left(\sum_{i=1}^{4} \delta_{i} e_{i}\right)$ and $\delta_{i}=0$ or 1 and $K=Z\left[e_{2}, e_{3}, e_{4}\right]$. Then, since $Z\left[e_{1}\right]$ and $K$ are maximal, $\delta_{1}=1$ holds and $\delta_{2}=\delta_{3}=\delta_{4}=0$ does not hold. By changing the basis of $K$, we may assume $u=\frac{1}{2}\left(e_{1}+e_{2}\right)$. From the evenness of $L$ follow $Q(u) \equiv 0 \bmod 2$ and $B\left(u, e_{3}\right) \equiv B\left(u, e_{4}\right) \equiv 0 \bmod 1$. Hence we have $Q\left(e_{2}\right) \equiv 6 \bmod 8, B\left(e_{2}, e_{3}\right) \equiv B\left(e_{2}, e_{4}\right) \equiv 0 \bmod 2$. Let $L^{\prime}$ be any maximal even quadratic lattice containing $Z\left[e_{1}\right] \perp K$; then as above we have $L^{\prime}=Z\left[e_{1}\right] \perp K+Z[v]$, where $v=\frac{1}{2}\left(e_{1}+\sum_{i=2}^{4} \delta_{i} e_{i}\right)$ and $\delta_{i}=0$ or 1 . From the evenness of $L^{\prime}$ follows $\delta_{4} B\left(e_{3}, e_{4}\right) \equiv \delta_{3} B\left(e_{3}, e_{4}\right) \equiv 0 \bmod 2$. Since $B\left(e_{3}, e_{4}\right)$ is odd, $\delta_{3}=\delta_{4}=0$. This implies $L=L^{\prime} . \quad Z\left[e_{1}\right]^{\perp}$ is obviously $K$.

In the following, $L(K)$ denotes a nice quaternary lattice constructed as above.

1.3. LEMma. Let $K$ be a nice ternary lattice. Then any element $x$ in $L(K)$ with $Q(x)=2$ is mapped on $\pm e_{1}$ by some symmetry of $L(K)$, or $x$ is already in $K$.

Proof. Using notation in 1.2, put $x=a e_{1}+b / 2\left(e_{1}+e_{2}\right)+c e_{3}+$ $d e_{4} \in L(K)$. Then we have

$$
Q,(x)=2\left(a+\frac{b}{2}\right)^{2}+Q\left(\frac{b}{2} e_{2}+c e_{3}+d e_{4}\right)=2 .
$$

If $b$ is odd, then $a+b / 2= \pm \frac{1}{2}$. Take $\delta= \pm 1$ satisfying $\delta-(a+b / 2)$ $= \pm \frac{1}{2}$. Then $\delta e_{1}-x$ is in $L(K), Q\left(\delta e_{1}-x\right)=2$ and $\tau_{\delta e_{1}-x}(x)=\delta e_{1}$. If $b$ is even, then $a+b / 2=0$ or \pm 1 . Hence $x$ is in $K$ or $x= \pm e_{1}$.

1.4. LEMma. Let $K_{1}, K_{2}$ be nice ternary lattices and suppose $Q\left(K_{2}\right)$ $\nexists 2$. If, then, $L\left(K_{1}\right)$ is isometric to $L\left(K_{2}\right)$, then $K_{1}$ is isometric to $\dot{K}_{2}$.

Proof. Let $\sigma$ be an isometry from $L\left(K_{1}\right)$ to $L\left(K_{2}\right)$. From our as- 
sumption and 1.3 we may assume $\sigma\left(e_{1}\right)=e_{1}$. On the other hand, $Z\left[e_{1}\right]^{\perp}$ in $L\left(K_{1}\right)$ is $K_{1}$ and $Z\left[e_{1}\right]^{\perp}$ in $L\left(K_{2}\right)$ is $K_{2}$. This implies $\sigma\left(K_{1}\right)=K_{2}$.

1.5. LEMMA. Let $K$ be a nice ternary lattice and let $K$ contain an element $e_{2}$ with $Q\left(e_{2}\right)=2$. Then $Z\left[e_{2}\right]^{\perp}$ is a nice binary lattice.

Proof. Since $K$ is indecomposable, $B\left(e_{2}, K\right) \neq 0 \bmod 2$. We take a basis $\left\{e_{2}, e_{3}, e_{4}\right\}$ of $K$ satisfying $B\left(e_{2}, e_{3}\right)=1, B\left(e_{2}, e_{4}\right)=0$; then $Z\left[e_{2}\right]^{\perp}=$ $Z\left[e_{2}-2 e_{3}, e_{4}\right]$ and $\left[K: Z\left[e_{2}\right] \perp Z\left[e_{2}\right]^{\perp}\right]=2$. This implies $d\left(Z\left[e_{2}\right]^{\perp}\right)=4 p$. It is easy to see that the Hasse invariant $S_{2}\left(Z\left[e_{2}\right]^{\perp}\right)=-\left(\frac{2}{p}\right)$. Hence $Z\left[e_{2}\right]^{\perp}$ is nice.

1.6. LEMMA. Let $M$ be a nice binary lattice. Then there are only two different even maximal ternary quadratic lattices containing $Z\left[e_{2}\right] \perp M$, $\left(Q\left(e_{2}\right)=2\right)$, and they are both nice.

Proof. From our assumption on $M$ follows $M \cong\langle 2 u\rangle \perp\left\langle 2 u^{-1} p\right\rangle$ over $Z_{2}$, where $u$ is a unit $\equiv 3 \bmod 4$ of $Z_{2}$. Since $\langle 2\rangle \perp\langle 2 u p\rangle$ is not maximal over $Z_{2}, Z\left[e_{2}\right] \perp M$ is not maximal. Let $K$ be any even maximal lattice containing $Z\left[e_{2}\right] \perp M$. Then we get $\left[K ; Z\left[e_{2}\right] \perp M\right]=2$ and $d(K)=2 p$. This means that $K$ is nice. Taking some basis $\left\{e_{3}, e_{4}\right\}$ of $M$, we may put $K=Z\left[e_{2}\right] \perp M+Z[v]$, where $v=\frac{1}{2}\left(e_{2}+e_{3}\right)$. The evenness of $K$ implies $Q\left(e_{3}\right) \equiv 6 \bmod 8, \quad B\left(e_{3}, e_{4}\right) \equiv 0 \bmod 2 . \quad$ Since $4 p=Q\left(e_{3}\right) Q\left(e_{4}\right)-$ $B\left(e_{3}, e_{4}\right)^{2}$, we have $Q\left(e_{4}\right) \equiv 4$ or $6 \bmod 8$. If $Q\left(e_{4}\right) \equiv 4 \bmod 8$, then $Q\left(e_{3}+e_{4}\right)$ $\equiv 6 \bmod 8$ and $M=Z\left[e_{3}, e_{3}+e_{4}\right]$. Without loss of generality we may assume $Q\left(e_{3}\right) \equiv Q\left(e_{4}\right) \equiv 6 \bmod 8$ and $B\left(e_{3}, e_{4}\right) \equiv 0 \bmod 4$. Let $K^{\prime}$ be any even maximal quadratic lattice containing $Z\left[e_{2}\right] \perp M$ and put $K^{\prime}=Z\left[e_{2}\right] \perp M$ $+Z[v]$, where $v=\frac{1}{2}\left(e_{2}+\delta_{3} e_{3}+\delta_{4} e_{4}\right)\left(\delta_{i}=0\right.$ or 1$)$. Then the evenness of $K^{\prime}$ implies either $\delta_{3}=1, \delta_{4}=0$ or $\delta_{3}=0, \delta_{4}=1$. Conversely $Z\left[e_{2}\right] \perp M$ $+Z\left[\frac{1}{2}\left(e_{2}+e_{4}\right)\right]$ is even and it is not equal to $Z\left[e_{2}\right] \perp M+Z\left[\frac{1}{2}\left(e_{2}+e_{3}\right)\right]$.

1.7. Lemma. Let $M$ be a nice binary lattice, and $K$ be an even maximal lattice containing $Z\left[e_{2}\right] \perp M,\left(Q\left(e_{2}\right)=2\right)$. Then any element $x$ in $K$ with $Q(x)=2$ is mapped on $\pm e_{2}$ by some symmetry of $K$.

Proof. If $Q(M) \ni 2$, then the Hasse invariant $S_{2}(M)$ of $M$ is $\left(\frac{2}{p}\right)$. Hence $Q(M) \nexists 2$. A quite similar method to the proof of lemma in 1.3 implies our statement. 
1.8. Lemma. Let $M_{1}, M_{2}$ be nice binary lattices and $K_{1}, K_{2}$ be corresponding nice ternary lattices constructed in 1.6. If, then, $K_{1}, K_{2}$ are isometric, then $M_{1}, M_{2}$ are isometric.

The proof is the same as 1.4 .

1.9. LEMMA. Let $M$ be a nice binary lattice and $K_{1}, K_{2}$ be different nice ternary lattices constructed from $M$ in 1.6. Then $K_{1}, K_{2}$ are isometric if and only if $M$ has a non-trivial isometry.

Proof. Let $M=Z\left[e_{3}, e_{4}\right]$ and $K_{1}=Z\left[e_{2}\right] \perp M+Z\left[\frac{1}{2}\left(e_{2}+e_{3}\right)\right], K_{2}=$ $Z\left[e_{2}\right] \perp M+Z\left[\frac{1}{2}\left(e_{2}+e_{4}\right)\right]$ as in 1.6. Suppose that $\sigma$ is an isometry from $K_{1}$ to $K_{2}$. Then without loss of generality we may assume $\sigma\left(e_{2}\right)=e_{2}$. Since $M=Z\left[e_{2}\right]^{\perp}$ in $K_{i}(i=1,2), \sigma$ induces an isometry of $M$. Hence we get $\sigma\left(\frac{1}{2}\left(e_{2}+e_{3}\right)\right) \equiv \frac{1}{2}\left(e_{2}+e_{4}\right) \bmod Z\left[e_{2}\right] \perp M$, and so $\sigma\left(e_{3}\right) \equiv e_{4} \bmod 2 M$. This shows that $\sigma$ is not \pm 1 on $M$. Conversely suppose that $M$ has a non-trivial isometry $\bar{\sigma}$, then there exists a basis $\{x, y\}$ of $\mathrm{M}$ satisfying $\bar{\sigma} x=y, \bar{\sigma} y=x$. If $K=Z\left[e_{2}\right] \perp M+Z\left[\frac{1}{2}\left(e_{2}+\delta_{1} x+\delta_{2} y\right)\right],\left(\delta_{i}=0\right.$ or 1$)$, is nice, then none of $\delta_{1}=\delta_{2}=0$ and $\delta_{1}=\delta_{2}=1$ can hold. Hence without loss of generality we may put $K_{1}=Z\left[e_{2}\right] \perp M+Z\left[\frac{1}{2}\left(e_{2}+x\right)\right], K_{2}=Z\left[e_{2}\right]$ $\perp M+Z\left[\frac{1}{2}\left(e_{2}+y\right)\right] . \quad \sigma$ satisfying $\sigma\left(e_{2}\right)=e_{2}, \sigma=\bar{\sigma}$ on $M$ gives an isometry from $K_{1}$ to $K_{2}$.

1.10. Lemma. Let $M_{i}$ be a nice binary lattice and $K_{i}$ be a nice ternary lattice constructed from $M_{i}$ in 1.6. If $L\left(K_{1}\right)$ and $L\left(K_{2}\right)$ are isometric, then $M_{1}$ and $M_{2}$ are isometric.

Proof. Let $\sigma$ be an isometry from $L\left(K_{1}\right)$ to $L\left(K_{2}\right)$. Using notation in 1.2, we may, by virtue of 1.3 , assume $\sigma\left(e_{1}\right)=e_{1}$ or $\sigma\left(e_{1}\right) \in K_{2}$. If $\sigma\left(e_{1}\right)$ $=e_{1}$, then $\sigma\left(K_{1}\right)=K_{2}$, and 1.8 implies that $M_{1}, M_{2}$ are isometric. If $\sigma\left(e_{1}\right) \in K_{2}$, then $M=Z\left[\sigma\left(e_{1}\right)\right]^{\perp}$ in $K_{2}$ is isometric to $M_{2}$ by virtue of 1.5 and 1.8. On the other hand, $K_{1}=Z\left[e_{1}\right]^{\perp}$ in $L\left(K_{1}\right)$ is isometric to $K^{\prime}=$ $Z\left[\sigma\left(e_{1}\right)\right]^{\perp}$ in $L\left(K_{2}\right)$, and $K^{\prime}$ is nice and contains $Z\left[e_{1}\right] \perp M$. Hence from 1.8 it follows that $M_{1}$ and $M$ are isometric, and so $M_{1}$ and $M_{2}$ are isometric.

1.11. Lemma. Let $M$ be a nice binary lattice, and $K_{1}, K_{2}$ be different nice ternary lattices constructed from $M$ in 1.6. Then $L\left(K_{1}\right)$ and $L\left(K_{2}\right)$ are different but isometric.

Proof. As in 1.6 we take a basis $\left\{e_{3}, e_{4}\right\}$ of $M$ such that $Q\left(e_{3}\right) \equiv$ 
$Q\left(e_{4}\right) \equiv 6 \bmod 8$ and $B\left(e_{3}, e_{4}\right) \equiv 0 \bmod 4$, and put $K_{1}=Z\left[e_{2}\right] \perp M+Z\left[\frac{1}{2}\left(e_{2}\right.\right.$ $\left.\left.+e_{3}\right)\right], K_{2}=Z\left[e_{2}\right] \perp M+Z\left[\frac{1}{2}\left(e_{2}+e_{4}\right)\right]$, where $Q\left(e_{2}\right)=2$. If $L\left(K_{1}\right)=L\left(K_{2}\right)$, then. $Z\left[e_{1}\right]^{\perp}$ in $L\left(K_{1}\right)$ and $Z\left[e_{1}\right]^{\perp}$ in $L\left(K_{2}\right)$ are equal and so $K_{1}=K_{2}$. This is a contradiction. Therefore we have $L\left(K_{1}\right) \neq L\left(K_{2}\right)$. We define an isometry $\sigma$ of $L\left(K_{1}\right) \otimes_{z} Q\left(=L\left(K_{2}\right) \otimes_{z} Q\right)$ by $\sigma\left(e_{1}\right)=e_{2}, \sigma\left(e_{2}\right)=e_{1}, \sigma\left(e_{3}\right)=e_{3}$, $\sigma\left(e_{4}\right)=e_{4}$. Firstly $\sigma\left(L\left(K_{1}\right)\right)$ contains $Z\left[e_{1}, e_{2}\right] \perp M$. Denote $\frac{1}{2}\left(e_{1}+e_{4}\right)$ by $v$. Then $Z\left[e_{1}\right] \perp K_{1}+Z[v]$ is even since $Q(v) \equiv 0 \bmod 2$ and $B\left(v, e_{1}\right) \equiv$ $B\left(v, e_{2}\right) \equiv B\left(v, e_{4}\right) \equiv B\left(v, \frac{1}{2}\left(e_{2}+e_{3}\right)\right) \equiv 0 \bmod 1$. Hence 1.2 implies $v \in L\left(K_{1}\right)$, and we have $\sigma\left(L\left(K_{1}\right)\right) \ni \sigma(v)=\frac{1}{2}\left(e_{2}+e_{4}\right)$. Since $\sigma\left(L\left(K_{1}\right)\right)$ is a nice quaternary lattice containing $Z\left[e_{1}\right] \perp K_{2}$, the uniqueness of $L\left(K_{2}\right)$ implies $\sigma\left(L\left(K_{1}\right)\right)=L\left(K_{2}\right)$.

1.12. Denote by $h(p)$ the class number of nice quaternary lattices and by $h^{+}(p)$ the class number with respect to rotations (i.e., $O^{+}$over $Q$ ) of nice quaternary lattices. Let $L$ be a nice quaternary lattice. If, then, any lattice which is isometric to $L$ is isometric to $L$ with respect to rotations, then $L$ has a refrexion $\sigma$ (i.e., an isometry with $\operatorname{det} \sigma=$ $-1)$. By virtue of $\sigma \neq \pm 1$, it follows from 2.5 in the next section that $Q(L) \ni 2$. Hence it follows from the above that

$$
\begin{aligned}
2 h(p) & -h^{+}(p) \\
= & \text { the class number of nice quaternary lattices which represent } 2 \\
= & \text { the class number of nice ternary lattices }-\frac{h(\sqrt{-p}) / 2-a}{2},
\end{aligned}
$$

where $h(\sqrt{-p})$ is the class number of $Q(\sqrt{-p})$ and $a$ stands for the number of ambigous classes contained in non-principal genus of $Q(\sqrt{-p})$, (hence necessarily $a=0$ or 1 ). On the other hand, the class number of nice ternary lattices is $\frac{1}{4}(h \sqrt{-p})+\frac{1}{24}\left(p+3-4\left(\frac{p}{3}\right)\right)$ due to Eisenstein and Mordell [2], [4].

Thus, we obtain

THEOREM. We have $2 h(p)-h^{+}(p)=\frac{1}{24}\left(p+3-4\left(\frac{p}{3}\right)\right)+a / 2$, where (-) stands for the quadratic residue symbol and $a$ is either 0 or 1 in order that the right hand be an integer, and $p$ is a prime $\equiv 1 \bmod 4$.

CoRollary. $h^{+}(p)$ is asymptotically equal to $2 h(p)$. 
Proof. From the simple calculation of the mass, it is easy to see $h(p)>c p^{3 / 2}$, where $c$ is a positive constant independent of $p$.

Remark 1. T. Tamagawa showed in the Summer Institute at Tokyo from June 29 to July 8, 1970, that $h^{+}(p)$ is the type number of some quaternion algebra over $Q(\sqrt{-p})$.

Remark 2. It is easy to generalize the above corollary to even positive definite lattices of an even dimension by the similar way to [3].

Remark 3. The number $a$ in the above theorem is zero if and only if $p \equiv 1 \bmod 8$.

Remark 4. The class number $2 h(p)-h^{+}(p)$ of nice quaternary lattices which represent two is a half of the dimension of the space $\mathfrak{M}\left(-2, \Gamma_{0}(p),\left(\frac{*}{p}\right)\right)$ of automorphic forms of dimension -2 and character $\left(\frac{*}{p}\right)$ with respect to $\Gamma_{0}(p)$. It is natural to ask whether $\vartheta(\tau, A)$ and $\vartheta\left(\tau, p A^{-1}\right)$ form a basis of $\mathfrak{M}\left(-2, \Gamma_{0}(p),\left(\frac{*}{p}\right)\right)$, where $A$ runs over representatives of classes of nice quaternary lattices which represent two. In case of $p \leq 109$, it is true.

\$. Let $N$ be a quadratic lattice over $Z$. Let $O(N)$ denote the orthogonal group of $N$, and let $S(N)$ denote the subgroup of $O(N)$ generated by symmetries of $N$ and \pm 1 . In general $S(N)$ is not equal to $O(N)$.

Our aim in this section is to prove

THEOREM. Let $L$ be a nice quaternary lattice. Then $O(L)=S(L)$.

2.1. Let $L$ be a nice quaternary lattice and $O(L)$ contain an element $\sigma$ of order 5 . Then by virtue of Th. 74.3 in [1] there exists a basis $\left\{e_{1}, e_{2}, e_{3}, e_{4}\right\}$ of $L$ such that

$$
\begin{aligned}
\sigma=\left(\begin{array}{cccc}
0 & 0 & 0 & -1 \\
1 & 0 & 0 & -1 \\
0 & 1 & 0 & -1 \\
0 & 0 & 1 & -1
\end{array}\right) \text { and }\left(B\left(e_{i}, e_{j}\right)\right) & \\
& =\left(\begin{array}{cccc}
2(b+c) & & \\
& -b & -c & -c \\
& 2(b+c) & -b & -c \\
& * & 2(b+c) & -b \\
& & 2(b+c)
\end{array}\right)
\end{aligned}
$$


Hence we have $p=\operatorname{det}\left(B\left(e_{i}, e_{j}\right)\right)=5\left(b^{2}+3 b c+c^{2}\right)^{2}$ and so $p=5$. In this case $L$ represents 2 .

-2.2. Let $L$ be a nice quaternary lattice and let $O(L)$ contain an element $\sigma$ of order 3 . Then one of the following cases holds.

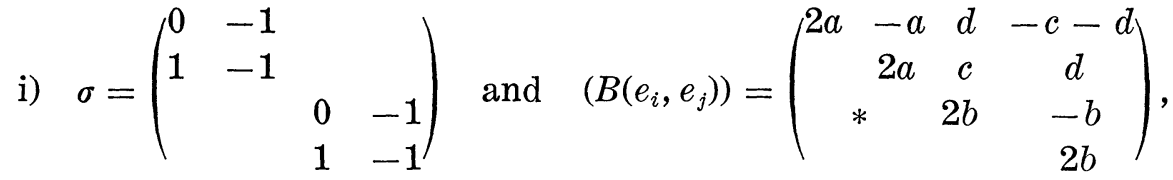

$$
\begin{aligned}
& \text { ii) } \sigma=\left(\begin{array}{llll}
0 & -1 & & \\
1 & -1 & & \\
& & 1 & 0 \\
& & 0 & 1
\end{array}\right) \text { and }\left(B\left(e_{i}, e_{j}\right)\right)=\left(\begin{array}{cccc}
* & 0 & 0 \\
0 & 0 & 0 & 0 \\
0 & 0 & *
\end{array}\right) \text {, } \\
& \text { iii) } \sigma=\left(\begin{array}{cccc}
0 & -1 & 1 & 0 \\
1 & -1 & 0 & 0 \\
0 & 0 & 1 & 0 \\
0 & 0 & 0 & 1
\end{array}\right) \text { and }\left(B\left(e_{i}, e_{j}\right)\right)=\left(\begin{array}{cccc}
2 a & -a & -a & 0 \\
2 a & 0 & 0 \\
* & b & d
\end{array}\right) \text {. }
\end{aligned}
$$

In case of i) $\operatorname{det}\left(B\left(e_{i}, e_{j}\right)\right)=\left(d^{2}+c^{2}+c d-3 a b\right)^{2}=p$. This is a contradiction. The case ii) contradicts $\operatorname{det}\left(B\left(e_{i}, e_{j}\right)\right)=p$, too. In case of iii) we want to show $a=1$. $\operatorname{det}\left(B\left(e_{i}, e_{j}\right)\right)=p$ implies $a=1$ or $p$. Suppose $a=p$. Then $Z\left[e_{1}\right]^{\perp}=Z\left[e_{1}+2 e_{2}, e_{2}-e_{3}, e_{4}\right]$ and $\left[L: Z\left[e_{1}\right] \perp Z\left[e_{1}\right]^{\perp}\right]$ $=2$. This implies that $Z\left[e_{1}\right]^{\perp}$ is an even ternary positive definite lattice of $d\left(Z\left[e_{1}\right]^{\perp}\right)=2$. But such a lattice does not exist. Hence $L$ represents 2 .

2.3. Let $L$ be a nice quaternary and $O(L)$ contain a non-trivial element $\sigma$ of order 2 . Then one of the following cases holds.

$$
\begin{aligned}
& \text { i) } \sigma=\left(\begin{array}{rrrr}
-1 & 1 & & \\
0 & 1 & & \\
& & 1 & 0 \\
& & 0 & 1
\end{array}\right) \text { and }\left(B\left(e_{i}, e_{j}\right)\right)=\left(\begin{array}{cccc}
2 a & -a & 0 & 0 \\
-a & & & \\
0 & & * & \\
0 & &
\end{array}\right) \text {, } \\
& \text { ii) } \sigma=\left(\begin{array}{cccc}
1 & & & \\
& 1 & & \\
& & -1 & \\
& & & -1
\end{array}\right) \text { and }\left(B\left(e_{i}, e_{j}\right)\right)=\left(\begin{array}{cccc}
* & 0 & 0 \\
& & 0 & 0 \\
0 & 0 & & \\
0 & 0 & *
\end{array}\right) \text {, }
\end{aligned}
$$

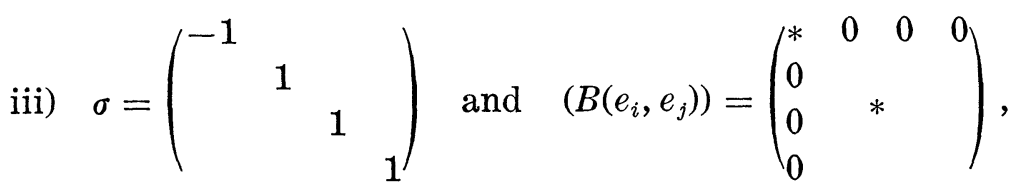



iv) $\sigma=\left(\begin{array}{llll}0 & 1 & & \\ 1 & 0 & & \\ & & 0 & 1 \\ & & 1 & 0\end{array}\right)$ and $\left(B\left(e_{i}, e_{j}\right)\right)=\left(\begin{array}{llll}a & b & e & f \\ b & a & f & e \\ e & f & c & d \\ f & e & d & c\end{array}\right)$,
v) $\sigma=\left(\begin{array}{cccc}0 & 1 & & \\ 1 & 0 & & \\ & & 1 & \\ & & & -1\end{array}\right)$ and $\left(B\left(e_{i}, e_{j}\right)\right)=\left(\begin{array}{cccc}a & b & e & f \\ b & a & e & -f \\ e & e & c & 0 \\ f & -f & 0 & d\end{array}\right)$.

The cases ii), iii) contradict $\operatorname{det}\left(B\left(e_{i}, e_{j}\right)\right)=p$. In case of $\mathrm{v}$ ), $\operatorname{det}\left(B\left(e_{i}, e_{j}\right)\right) \equiv 0 \bmod 2$. This is also a contradiction. In case of $\left.\mathrm{i}\right), a=1$ is proved by the same way as the proof of the case iii) in 2.2. In case of iv) $p=\operatorname{det}\left(B\left(e_{i}, e_{j}\right)\right)=\left\{(a-b)(c-d)-(e-f)^{2}\right\}\left\{(a+b)(c+d)-(e+f)^{2}\right\}$. Put $N_{+}=Z\left[e_{1}+e_{2}, e_{3}+e_{4}\right], N_{-}=Z\left[e_{1}-e_{2}, e_{3}-e_{4}\right]$, then

$$
\begin{aligned}
& d\left(N_{+}\right)=4\left\{(a+b)(c+d)-(e+f)^{2}\right\}, \\
& d\left(N_{-}\right)=4\left\{(a-b)(c-d)-(e-f)^{2}\right\} .
\end{aligned}
$$

Hence $N_{+}$or $N_{-}$is an even binary lattice of discriminant 4 , and it represents 2 .

2.4. Let $L$ be a nice quaternary lattice and $O(L) \neq\{ \pm 1\}$. Suppose that $O(L)$ does not contain isometries of order 3 or 5 , nor non-trivial isometries of order 2. Then $O(L)$ contains an element $\sigma$ of order 4 and $\sigma^{2}=-1 . \quad Z[\sigma]$ is isomorphic to $Z[\sqrt{-1}]$ and $Z[\sigma]$ operates torsion-freely on $L$. Hence we may assume

$$
\sigma=\left(\begin{array}{cccc}
0 & -1 & & \\
1 & 0 & & \\
& & 0 & -1
\end{array}\right) \text { and } \quad\left(B\left(e_{i}, e_{j}\right)\right)=\left(\begin{array}{cccc}
a & 0 & c & d \\
0 & a & -d & c \\
c & -d & b & 0 \\
d & c & 0 & b
\end{array}\right) .
$$

This is a contradiction, because $p=\operatorname{det}\left(B\left(e_{i}, e_{j}\right)\right)=\left(a b-c^{2}-d^{2}\right)^{2}$.

2.5. Summing up: let $L$ be a nice quaternary lattice. If $O(L) \neq\{ \pm 1\}$, then $Q(L) \ni 2$.

2.6. Lemma. Let $K$ be a nice ternary lattice. If $O(K) \neq\{ \pm 1\}$, then $Q(K) \ni 2$.

Proof. As above we may assume that $O(K)$ contains an isometry $\sigma$ which is one of the following form for some basis $\left\{v_{1}, v_{2}, v_{3}\right\}$ of $K$ : 

i) $\left(\begin{array}{lll}0 & -1 & \\ 1 & -1 & \\ & & 1\end{array}\right)$,
ii) $\left(\begin{array}{ccc}0 & -1 & 1 \\ 1 & -1 & 0 \\ 0 & 0 & 1\end{array}\right)$,
iii) $\left(\begin{array}{ccc}-1 & 1 & \\ 0 & 1 & \\ & & 1\end{array}\right)$,
iv) $\left(\begin{array}{lll}1 & & \\ & 1 & \\ & & -1\end{array}\right)$.

In case of $i), i v), K$ is decomposable. This is a contradiction. In case of ii) we have

$$
\left(B\left(v_{i}, v_{j}\right)\right)=\left(\begin{array}{ccc}
2 a & -a & -a \\
-a & 2 a & 0 \\
-a & 0 & b
\end{array}\right)
$$

Since $d(K)=\operatorname{det}\left(B\left(v_{i}, v_{j}\right)\right)=2 p$, we have $a=1$.

In case of iii), we have

$$
\left(B\left(v_{i}, v_{j}\right)\right)=\left(\begin{array}{ccc}
2 a & -a & 0 \\
-a & b & e \\
0 & e & c
\end{array}\right),
$$

and $\operatorname{det}\left(B\left(v_{i}, v_{j}\right)\right)=2 p$ implies $a=1$ or $p$. If $a=1$, then $Q(K) \ni 2$. If $a=p$, then $d\left(Z\left[v_{1}+2 v_{2}, v_{3}\right]\right)=4$. From this follows $Q(K) \ni 2$.

2.7. By virtue of 2.5 we may, to prove our theorem, assume that a nice quaternary lattice $L$ is either $L(K)$, where $K$ is a nice ternary lattice and $Q(K) \nexists 2$, or a nice quaternary lattice constructed from a nice binary lattice $M$ as in 1.11 .

Firstly suppose that $L=L(K)$ and $Q(K) \not 2$. Take any $\sigma$ in $O(L)$. From $Q(K) \not 2$ and 1.3 (the meaning of $e_{1}$ remains same) follows $\sigma\left(e_{1}\right)=$ $\tau\left(e_{1}\right)$ for some $\tau$ in $S(L)$, and $\tau^{-1} \sigma\left(e_{1}\right)=e_{1}$. Since $Z\left[e_{1}\right]^{\perp}$ is equal to $K, \tau^{-1} \sigma$ induces an isometry of $K$, and 2.6 implies that $\tau^{-1} \sigma= \pm 1$ on $K$. Hence we get $\tau^{-1} \sigma=1$ or $-\tau_{e_{1}}$. This shows $\sigma \in S(L)$.

2.8. Suppose that $L$ is a lattice constructed as 1.11 . Without loss of generality, we may assume $L=L\left(K_{1}\right)$ in 1.11. Using symbols in 1.11, we show $\alpha \in S(L)$ for any $\alpha$ in $O(L)$. If $\alpha\left(e_{1}\right) \notin K_{1}$, then we may assume $\alpha\left(e_{1}\right)=e_{1}$ by virtue of 1.3. This implies $\alpha\left(K_{1}\right)=K_{1}$. From 1.7 follows that there exists some $\bar{\tau} \in S\left(K_{1}\right)$ such that $\bar{\tau} e_{2}=\alpha e_{2}$. Since a nice quaternary lattice containing $Z\left[e_{1}\right] \perp K_{1}$ is unique, $\tau$ satisfying $\left.\tau\right|_{K_{1}}=\bar{\tau}$ and $\tau\left(e_{1}\right)=e_{1}$ is in $S(L)$. Hence $\tau^{-1} \alpha$ satisfies $\tau^{-1} \alpha\left(e_{1}\right)=e_{1}, \tau^{-1} \alpha\left(e_{2}\right)=e_{2}$. Since $M=$ 
$Z\left[e_{1}, e_{2}\right]^{\perp}$, we get $\tau^{-1} \alpha(M)=M$. The relations $\tau^{-1} \alpha\left(K_{1}\right)=K_{1}$ and $\tau^{-1} \alpha(M)$ $=M$ imply $\tau^{-1} \alpha\left(\frac{1}{2}\left(e_{2}+e_{3}\right)\right) \equiv \frac{1}{2}\left(e_{2}+e_{3}\right) \bmod Z\left[e_{2}\right] \perp M$ and so $\tau^{-1} \alpha\left(e_{3}\right) \equiv e_{3}$ $\bmod 2 M$. This means that $\tau^{-1} \alpha$ induces a trivial isometry \pm 1 on $M$. For, otherwise, as in the proof of lemma in 1.9 we may assume $\tau^{-1} \alpha\left(e_{3}\right)$ $=e_{4}$. Hence we have $\tau^{-1} \alpha=\mathrm{id}$. or $-\tau_{e_{1}} \tau_{e_{2}} \in S(L)$.

Suppose $\alpha\left(e_{1}\right) \in K_{1}$. By virtue of 1.7 we assume $\alpha\left(e_{1}\right)=e_{2}$. Since $\alpha\left(K_{1}\right)=Z\left[e_{2}\right]^{\perp}($ in $L), B\left(e_{1}, e_{2}\right)=B\left(e_{2}, \alpha\left(e_{2}\right)\right)=0$, we may assume moreover $\alpha\left(e_{2}\right)=e_{1}$, applying 1.7 to $\alpha\left(K_{1}\right)$. Then we have $\alpha(M)=M$ again, and $\frac{1}{4} B\left(e_{3}, \alpha\left(e_{3}\right)\right) \equiv 0 \bmod 1$, because $B\left(\frac{1}{2}\left(e_{2}+e_{3}\right), \alpha\left(\frac{1}{2}\left(e_{2}+e_{3}\right)\right)\right)$ is an integer. This shows that $\alpha$ is not \pm 1 on $M$, since $Q\left(e_{3}\right) \equiv 6 \bmod 8$. Without loss of generality we may take a basis $\left\{e_{3}, e_{4}\right\}$ of $M$ satisfying $\alpha\left(e_{3}\right)=$ $e_{4}, \alpha\left(e_{4}\right)=e_{3}$ as in the proof of lemma in 1.9 , and $M \cong\left(B\left(e_{i}, e_{j}\right)\right)$ $=\left(\begin{array}{ll}p+1 & p-1 \\ p-1 & p+1\end{array}\right) . \quad$ Put $x=\frac{1}{2}\left(-e_{1}+e_{2}+e_{3}-e_{4}\right)$; then $x$ is in $L$, since $Z\left[e_{1}\right] \perp K+Z[x]$ is even. $Q\left(x-e_{2}\right)=2$ and $Q\left(x+e_{1}\right)=2$ imply $\tau_{x-e_{2}}$, $\tau_{x+e_{1}} \in S(L)$ and so $-\tau_{x+e_{1}} \tau_{x-e_{2}} \alpha\left(e_{1}\right)=e_{1}$. From the results in the first half of this section follows $-\tau_{x+e_{1}} \tau_{x-e_{2}} \alpha \in S(L)$. This completes the proof of our theorem.

§. In this section we calculate explicitely the class number $h(p)$ in 1.12 by method due to Eisenstein and Mordell [2], [4] assuming $p>5$ $(h(5)=1$ is known).

3.1. If $K$ is a nice ternary lattice, $Q(K) \not 2$, and $K$ does not contain any element $x$ satisfying $Q(x)=6, B(x, K) \equiv 0 \bmod 2$, then it follows from the proof of lemma in 1.3 that $x \in L(K)$ and $Q(x)=2$ imply $x= \pm e_{1}$ (using the notation there). Hence we have $|O(L)|=4$.

3.2. By definition $N$ is called an $E$-lattice if and only if $N$ is an even binary positive definite quadratic lattice such that the discriminant $d(N)=3 \mathrm{p}$ and the Hasse invariant $S_{2}(N)$ (resp. $S_{p}(N)$ ) at the prime 2 (resp. p) is $\left(\frac{2}{p}\right)\left(\right.$ resp. $\left.-\left(\frac{6}{p}\right)\right)$. In this case the Hasse invariant $S_{3}(N)$ is $-\left(\frac{3}{p}\right)$.

3.3. LEMma. Let $K$ be a nice ternary lattice such that $Q(K) \nexists 2$ and suppose that there is an element $x$ with $Q(x)=6, B(x, K) \equiv 0 \bmod 2$. Then $Z[x]^{\perp}$ is an E-lattice with $Q\left(Z[x]^{\perp}\right) \nexists 2,12$. 
Proof. Take a basis $\left\{x, e_{3}, e_{4}\right\}$ of $K$. Then $Q(x)=6, B\left(x, e_{3}\right) \equiv B\left(x, e_{4}\right)$ $\equiv 0 \bmod 2$ follow from our assumption. Without loss of generality we may- assume $B\left(x, e_{4}\right)=0$. Since $Z[x]^{\perp}=Z\left[3 e_{3}-\left(B\left(x, e_{3}\right) / 2\right) x, e_{4}\right]$ and $\left[K: Z[x] \perp Z[x]^{\perp}\right]=3$, we get $d\left(Z[x]^{\perp}\right)=3 p$, and the simple calculation of Hasse invariants shows that $Z[x]^{\perp}$ is an $E$-lattice. $Q\left(Z[x]^{\perp}\right) \not \nexists 2$ is obvious. Put $y=3 e_{3}-\left(B\left(x, e_{3}\right) / 2\right) x$; then $Q(y) \equiv B\left(y, e_{4}\right) \equiv 0 \bmod 3$. Suppose $Q\left(\ell y+m e_{4}\right)=12$ for some integers $\ell, m$. Since $d\left(Z[x]^{\perp}\right)=3 p$ implies $Q\left(e_{4}\right) \neq 0 \bmod 3$, we get $m \equiv 0 \bmod 3$ and $\ell \neq 0 \bmod 3$. Take an integer $a$ such that $a+\ell / 3= \pm \frac{1}{3}$; then either $a x+\ell / 3(x+y)+(m / 3) e_{4}$ or $a x+\ell / 3(x-y)-(m / 3) e_{4}$ is in $K$ and its norm is 2 . This is a contradiction.

3.4. LEMMA. Let $N$ be an E-lattice with $Q(N) \nexists 2,12$ and $K$ be a maximal even ternary lattice containing $Z\left[f_{2}\right] \perp N$, where $Q\left(f_{2}\right)=6$. Then $K$ is nice with $Q(K) \nexists 2$ and contains an element $x$ satisfying $Q(x)=6$, $B(x, K) \equiv 0 \bmod 2$, which is unique up to \pm .

Proof. Let $N \cong\langle u\rangle \perp\langle 3 p u\rangle$ over $Z_{3}$, where $u$ is a unit of $Z_{3}$; then we have $u \equiv p \bmod 3$, since $S_{3}(N)=-\left(\frac{u}{3}\right)=-\left(\frac{p}{3}\right)$. This implies that $Z_{3}\left[f_{2}\right] \perp\langle 3 p u\rangle=\langle 6\rangle \perp\langle 3\rangle$ is not maximal over $Z_{3}$. Hence $Z\left[f_{2}\right] \perp N$ is not maximal, and therefore $\left[K: Z\left[f_{2}\right] \perp N\right]=3$. This shows that $K$ is nice. For some basis $\left\{f_{3}, f_{4}\right\}$ of $N$ we have $K=Z\left[f_{2}\right] \perp N+Z\left[\frac{1}{3}\left(f_{2}+f_{3}\right)\right]$. The evenness of $K$ implies $Q\left(f_{3}\right) \equiv 12 \bmod 18$ and $B\left(f_{3}, f_{4}\right) \equiv 0 \bmod 3$. $Q(K) \nexists 2$ follows from $Q(N) \nexists 2,12$, and $f_{2}$ satisfies $Q\left(f_{2}\right)=6, B\left(f_{2}, K\right) \equiv 0$ $\bmod 2$. Conversely, let $v=a f_{2}+b / 3\left(f_{2}+f_{3}\right)+c f_{4}$ satisfy $Q(v)=6$, $B(v, K) \equiv 0 \bmod 2$. If $b \equiv 0 \bmod 3$, then $a+b / 3=0$ or \pm 1 . If $a+b / 3$ $= \pm 1$, then $v= \pm f_{2}$. If $a+b / 3=0$, then $Q(N) \ni 6$ and $S_{p}(N)=$ $(6,3 p)_{p}(6 \cdot 3 p,-1)_{p}=(6, p)_{p}$. This is a contradiction. Suppose $b \neq 0 \bmod 3$, then $|a+b / 3|=\frac{1}{3}$ or $\frac{2}{3}$. If $|a+b / 3|=\frac{1}{3}$ (resp. $\left.\frac{2}{3}\right)$, then $Q\left(b f_{3}+3 c f_{4}\right)$ $=48($ resp. 30$)$. From $B(v, K) \equiv 0 \bmod 2$ follows $B\left(v, f_{4}\right) \equiv B\left(v, \frac{1}{3}\left(f_{2}+f_{3}\right)\right)$ $\equiv 0 \bmod 2$, and we get $b \equiv c \equiv 0 \bmod 2$. This is a contradiction.

3.5. Lemma. Let $N_{1}, N_{2}$ be E-lattices with $Q\left(N_{i}\right) \not 2$, 12, and $K_{1}$, $K_{2}$ are corresponding nice ternary lattices constructed in 3.4. Then $N_{1}, N_{2}$ are isometric if and only if $L\left(K_{1}\right), L\left(K_{2}\right)$ are isometric.

Proof. Suppose that $N_{1}, N_{2}$ are isometric; then we may put $N_{1}=$ $N_{2}=N$. Let $f_{2}, f_{3}, f_{4}$ be as in the proof of lemma in 3.4. Put $K_{1}=$ 
$Z\left[f_{2}\right] \perp N+Z\left[\frac{1}{3}\left(f_{2}+f_{3}\right)\right]$, and $K_{2}=Z\left[f_{2}\right] \perp N+Z\left[\frac{1}{3}\left(f_{2}+\delta_{3} f_{3}+\delta_{4} f_{4}\right)\right]$, where $\delta_{3}, \delta_{4}=0$ or \pm 1 . From the evenness of $K_{2}$ follow then $\delta_{4}=0, \delta_{3}$ $= \pm 1$. Let $\sigma$ be an isometry of $\left(Z\left[f_{2}\right] \perp N\right) \otimes Q$ satisfying $\sigma\left(f_{2}\right)=f_{2}$, $\left.\sigma\right|_{N}=\delta_{3} \cdot$ id ; then $\sigma\left(K_{1}\right)=K_{2}$. This implies that $L\left(K_{1}\right), L\left(K_{2}\right)$ are isometric. Conversely suppose that $L\left(K_{1}\right), L\left(K_{2}\right)$ are isometric, then $K_{1}, K_{2}$ are isometric by virtue of 1.4, and 3.4 implies that $N_{1}, N_{2}$ are isometric.

3.6. LemMA. Let $K$ be a nice ternary lattice with $Q(K) \nexists 2$ such that $K$ contains an element $x$ satisfying $Q(x)=6, B(x, K) \equiv 0 \bmod 2$. Then $|O(L(K))|=12$.

Proof. Without loss of generality, we may assume that $K=Z\left[f_{2}\right]$ $\perp N+Z\left[\frac{1}{3}\left(f_{2}+f_{3}\right)\right]$, where $N=Z\left[f_{3}, f_{4}\right]$ is an $E$-lattice and $Q(N) \nexists 2,12$, $Q\left(f_{2}\right)=6, Q\left(f_{3}\right) \equiv 12 \bmod 18$ and $B\left(f_{3}, f_{4}\right) \equiv 0 \bmod 3$. Then we have $L(K)=Z\left[e_{1}\right] \perp K+Z\left[\frac{1}{2}\left(e_{1}+f_{2}\right)\right]$, where $Q\left(e_{1}\right)=2$. Let $x=a e_{1}+b / 2$ $\left(e_{1}+f_{2}\right)+c / 3\left(f_{2}+f_{3}\right)+d f_{4}$ be an element in $L(K)$ with $Q(x)=2$; then $2(a+b / 2)^{2}+6(b / 2+c / 3)^{2}+Q\left((c / 3) f_{3}+d f_{4}\right)=2$. If $b$ is even, then $a+b / 2=0$ or \pm 1 . In case of $a+b / 2=0, x$ is in $K$. This is a contradiction. In case of $a+b / 2= \pm 1$, we have $x= \pm e_{1}$. If $b$ is odd, then $a+b / 2= \pm \frac{1}{2}$ and $3 b+2 c= \pm 1$ or \pm 3 . In case of $3 b+2 c= \pm 1$, we have $Q\left(c f_{3}+3 d f_{4}\right)=12$. This is a contradiction. In case of $3 b+$ $2 c= \pm 3$, we have $x= \pm \frac{1}{2}\left(e_{1} \pm f_{2}\right)$. Hence we get $|O(L)|=12$.

3.7. Lemma. There is just one E-lattice $N$ with $Q(N) \ni 2$ if and only if $p \equiv-1 \bmod 3$.

Proof. Suppose $Q(N) \ni 2$, and take a basis $\{x, y\}$ of $N$ satisfying $Q(x)=2$. Since $d(N)=3 p$ implies $B(x, y) \equiv 1 \bmod 2$, we may assume $B(x, y)=1$, so that $Q(y)=(3 p+1) / 2$. This implies the uniqueness. On the other hand the Hasse invariant $S_{3}(N)$ is $(2,3 p)_{3}(6 p,-1)_{3}=1=-\left(\frac{p}{3}\right)$. Hence $p \equiv-1 \bmod 3$. Conversely assume $p \equiv-1 \bmod 3$, and put $N=$ $Z[x, y]$, where $Q(x)=2, B(x, y)=1, Q(y)=(3 p+1) / 2$; then $N$ is an $E$-lattice.

3.8. LEMma. There is just one E-lattice $N$ with $Q(N) \ni 12$ if and only if $p \equiv 5 \bmod 8$, and in this case $Q(N) \not 2$ if $p \equiv-1 \bmod 3$.

Proof. Suppose $Q(N) \ni 12$; then $S_{2}(N)=-1$. This implies $p \equiv 5$ $\bmod 8$. Conversely if $p \equiv 5 \bmod 8$, then $N \cong\left(\begin{array}{cc}12 & 3 \\ 3 & (p+3) / 4\end{array}\right)$ is an $E$ - 
lattice and $Q(N) \not 2$ if $p \equiv-1 \bmod 3$. (In this section, $p>5$ is assumed). The uniqueness is easy to prove.

3.9. LEMMA. The number (up to isometry) of E-lattices with $Q(N)$ $\not 2,12$ is $h(\sqrt{-3 p}) / 4+a^{\prime} / 2-\delta$, where $h(\sqrt{-3 p})$ is the ideal class number of $Q(\sqrt{-3 p})$, and

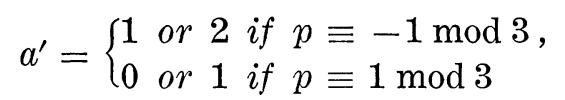

so that $h(\sqrt{-3 p}) / 4+a^{\prime} / 2$ is an integer, and

$$
\delta=\left\{\begin{array}{llr}
0 & \text { if } p \equiv 1 \\
1 & \text { if } p \equiv 13 \text { or } & 17 \bmod 24, \\
2 & \text { if } p \equiv 5 & \bmod 24
\end{array}\right.
$$

Proof. An $E$-lattice $N$ is in the principal genus if and only if $p \equiv$ $-1 \bmod 3$, then $a^{\prime}=1$ or $2\left(\alpha^{\prime}\right.$ is the number of ambigous classes in the genus of $N$ ).

3.10. Let $M$ be a nice binary lattice, take a basis $\left\{e_{3}, e_{4}\right\}$ of $M$ satisfying $Q\left(e_{3}\right) \equiv Q\left(e_{4}\right) \equiv 6 \bmod 8$ and $B\left(e_{3}, e_{4}\right) \equiv 0 \bmod 4$ as in the proof of 1.6, and put $K=Z\left[e_{2}\right] \perp M+Z\left[\frac{1}{2}\left(e_{2}+e_{3}\right)\right]$, where $Q\left(e_{2}\right)=2$; then $L=L(K)=Z\left[e_{1}\right] \perp K+Z\left[\frac{1}{2}\left(e_{1}+e_{4}\right)\right]$, where $Q\left(e_{1}\right)=2$. If $Q(M) \nexists 4,6$, then it is easy to see that $x= \pm e_{1}$ or $\pm e_{2}$ if $x \in L$ and $Q(x)=2$, and we get $|O(L)|=8$.

If $Q(M) \ni 4$, then $M \cong\left(\begin{array}{cc}4 & 2 \\ 2 & p+1\end{array}\right)$, and a simple calculation of $S_{2}(M)$ shows $p \equiv 5 \bmod 8$. Conversely if $p \equiv 5 \bmod 8$, then $M=Z[x, y]$, where $Q(x)=4, B(x, y)=2$ and $Q(y)=p+1$, is a nice binary lattice with $Q(M) \nexists 6$. Put $e_{3}=x+y, e_{4}=y$; then $M=Z\left[e_{3}, e_{4}\right]$, and $Q\left(e_{3}\right) \equiv Q\left(e_{4}\right) \equiv$ $6 \bmod 8$ and $B\left(e_{3}, e_{4}\right) \equiv 0 \bmod 4$ are satisfied. If, then, $x$ is an element of $L$ with $Q(x)=2$, then we have $x= \pm e_{1}, \pm e_{2}$ or $\pm\left\{a e_{1}+b e_{2}-\frac{1}{2}\left(e_{1}+e_{4}\right)\right.$ $\left.+\frac{1}{2}\left(e_{2}+e_{3}\right)\right\}$, where $\left|a-\frac{1}{2}\right|=\frac{1}{2},\left|b+\frac{1}{2}\right|=\frac{1}{2}$. Hence $|O(L)|=48$.

If $Q(M) \ni 6$, and if $\{x, y\}$ is a basis of $M$ with $Q(x)=6$, then we get $p \equiv-1 \bmod 3$ since $4 p=6 Q(y)-B(x, y)^{2}$. Conversely if $p \equiv-1$ $\bmod 3$, then, putting we have $Q\left(e_{3}\right)=6, Q\left(e_{4}\right)=(2 p+8) / 3, B\left(e_{3}, e_{4}\right)=4$, and $M=Z\left[e_{3}, e_{4}\right]$. Clearly this $M$ is nice. Therefore $x= \pm e_{1}, \pm e_{2}$ or $\pm \frac{1}{2}\left(e_{2} \pm e_{3}\right)$ if $x \in L$ and $Q(x)=2$. Thus, we get $|O(L)|=24$.

3.11. Nice quaternary lattices are in the same genus, and its mass 
is $\sum 1 /|O(L)|=B_{2, \chi} / 3 \cdot 2^{6}$, ( $L$; nice quaternary), where $B_{2, \chi}$ is a generalized Bernoulli number equal to $p \sum_{a=1}^{p} \chi(a) B_{2}(a-p / p)=4 /(7+2 \chi(2)) \sum_{a=1}^{(p-1) / 4}$ $\chi(a)(p-4 a)$ with $\chi(n)=\left(\frac{n}{p}\right)$.

3.12. Summing up the above results, we obtain

$$
\begin{aligned}
\frac{1}{2} h(p)= & \frac{B_{2, \chi}}{3 \cdot 2^{6}} \\
& +\left(\frac{1}{2}-\frac{1}{4}\right)\left\{\frac{1}{4} h(\sqrt{-p})+\frac{1}{24}\left(p+3-4\left(\frac{p}{3}\right)\right)-\frac{1}{2} h(\sqrt{-p})\right. \\
& \left.\quad-\left(\frac{1}{4} h(\sqrt{-3 p})+\frac{a^{\prime}}{2}-\delta\right)\right\} \\
& +\left(\frac{1}{2}-\frac{1}{12}\right)\left(\frac{1}{4} h(\sqrt{-3 p})+\frac{a^{\prime}}{2}-\delta\right) \\
& +\left(\frac{1}{2}-\frac{1}{8}\right)\left(\frac{1}{4} h(\sqrt{-p})+\frac{a}{2}-\delta_{1}-\delta_{2}\right) \\
& +\left(\frac{1}{2}-\frac{1}{48}\right) \delta_{1}+\left(\frac{1}{2}-\frac{1}{24}\right) \delta_{2},
\end{aligned}
$$

where

$$
\begin{aligned}
& \delta_{1}=\left\{\begin{array}{l}
0 \text { if } p \equiv 1 \bmod 8, \\
1 \text { if } p \equiv 5 \bmod 8,
\end{array} \delta_{2}=\left\{\begin{array}{l}
0 \text { if } p \equiv 1 \bmod 3, \\
1 \text { if } p \equiv-1 \bmod 3,
\end{array}\right.\right. \\
& a^{\prime}=\left\{\begin{array}{lll}
0 \text { or } 1 \text { if } p \equiv 1 \bmod 3 \\
1 \text { or } 2 \text { if } p \equiv-1 \bmod 3
\end{array}\right.
\end{aligned}
$$

in order that $\frac{1}{4} h(\sqrt{-3 p})+a^{\prime} / 2$ be an integer,

$$
\delta= \begin{cases}0 \text { if } p \equiv 1 \\ 1 \text { if } p \equiv 13 \text { or } 17 \bmod 24, \\ 2 \text { if } p \equiv 5 & \bmod 24,\end{cases}
$$

$a=0$ or 1 , in order that $\frac{1}{4} h(\sqrt{-p})+a / 2$ be an integer, and $h(\sqrt{-p})$ (resp. $h(\sqrt{-3 p})$ ) is the class number of $Q(\sqrt{-p})(\operatorname{resp} . Q(\sqrt{-3 p})$ ). In other words, we have

THEOREM. The class number $h(p)$ of even quaternary positive definite quadratic lattices of prime discriminant $p(\equiv 1 \bmod 4)>5$ is

$$
\frac{B_{2, x}}{2^{5} \cdot 3}+\frac{h(\sqrt{-p})}{16}+\frac{h(\sqrt{-3 p})}{12}+\frac{1}{48}\left(p+9-4\left(\frac{p}{3}\right)-6\left(\frac{p}{2}\right)\right) .
$$


The proper class number $h^{+}(p)$ in 1.12 is explicitely given by this theorem and the theorem in 1.12, and it was given in [7].

\section{REFERENCES}

[1] C. W. Curtis and I. Reiner, Representation theory of finite groups and associative algebras, Interscience Pub., 1962.

[2] G. Eisenstein, Tabelle der reducirten positive ternären quadratischen Formen, J. für Math., 41 (1851), 141-190.

[ 3 ] Y. Kitaoka, Two theorems on the class number of positive definite quadratic forms, Nagoya Math. J., 51 (1973), 79-89.

[4] L. J. Mordell, On the class number for definite ternary quadratics, Messenger of Math., 47 (1918), 65-78.

[ 5 ] O. T. O'Meara, Introduction to quadratic forms, Springer-Verlag, 1963.

[6] H. Pfeuffer, Einklassige Geschlechter totalpositiver quadratischer Formen in totalreellen algebraischen Zahlkörpern, J. number theory, 3 (1971), 371-411.

[ 7 ] P. Ponomarev, Class number of definite quaternary forms with nonsquare discriminant, Bull. Amer. Math. Soc., 79 (1973), 594-598.

[ 8 ] C. L. Siegel, Über die analytische Theorie der quadratischen Formen, Ann. Math., 36 (1935), 527-606.

Department of Mathematics

Nagoya University 